\title{
Structures Agro-Industrielles, Question Foncière Rurale Et Dynamiques Socioéconomiques Dans Le Sud Ivoirien
}

\author{
Toh Alain \\ Socio-anthropologue des questions foncières rurales \\ Université Félix Houphouët-Boigny d'Abidjan , Laboratoire d'Etudes et de \\ Recherches Interdisciplinaires en Sciences Sociales \\ Kone Lassina \\ Doctorant en Sociologie du développement rural \\ Université Félix Houphouët-Boigny d'Abidjan, Laboratoire d'Etudes et de \\ Recherches Interdisciplinaires en Sciences Sociales
}

doi: 10.19044/esj.2016.v13n2p196 URL:http://dx.doi.org/10.19044/esj.2016.v13n2p196

\begin{abstract}
This research examines the actions of agribusiness structures for populations and analyze the drivers of conflict between the structures and indigenous. The purposive sampling was used to select the various stakeholders in connection with the issue (youth, beneficiaries of the device and the elderly guardians of ancestral values) A total of 30 interviews (10 with youth opposed the succession device, 155 recipients and seniors) were carried out from September 2 to November 2, 2015 intermittently. This methodological approach has allowed us to achieve the following results: i) the balance of socio-economic development, ii) the inadequacy in the management of interests related to land and iii) the conflicts inherent in the presence of agroindustrial structures. However, is it appropriate to point out that agribusiness structures pay a fee to people so that they support.
\end{abstract}

Keywords: Dynamic land, agro-industrial structures, conflicts, socioeconomic issues

Résumé

Cette recherche examine les actions menées par les structures agroindustrielles en faveur des populations et analyser les facteurs de conflit entre les structures et les autochtones. L'échantillonnage par choix raisonné a permis de sélectionner les différents acteurs en lien avec la question (jeunes, bénéficiaires du dispositif et les personnes âgées gardiennes des valeurs ancestrales) Au total, 30 entretiens (10 avec les 
jeunes opposés au dispositif de succession, 15 bénéficiaires et 5 personnes âgées) ont été réalisés du 2 septembre au 2 novembre 2015 de façon discontinue. Cette démarche méthodologique nous a permis d'aboutir aux résultats suivants: i) le bilan du développement socio-économique, ii) l'inadéquation dans la gestion des intérêts liés au foncier et iii) les conflits inhérents à la présence des structures agroindustrielles. Toutefois, convientil de souligner que les structures agroindustrielles versent une redevance aux populations pour qu'elles se prennent en charge.

Mots-clés : Dynamiques foncières, structures agro-industrielles, Conflits, enjeux socio-économiques

\section{Introduction}

Après son accession à l'indépendance en 1960, la Côte d'Ivoire a opté pour le libéralisme économique, avec une grande marge de manœuvre accordée à l'initiative privée et à l'ouverture de son économie aux capitaux et échanges extérieurs (Sawadogo, 1977). Ainsi, de 1960 à 1970, les autorités ivoiriennes ont mis en place une politique d'industrialisation en focalisant leur vision de développement sur la création des structures de transformation en campagne axées sur les produits agricoles tels que le café, le cacao, le palmier à huile et l’hévéa (Toh, 2011).

Dans l'ensemble, cette politique d'industrialisation a contribué à la création de 110 entreprises en 1960, puis de 705 entreprises en 1980, soit une progression de $9 \%$ par an, avec des investissements industriels qui sont passés de 22 milliards de francs CFA en 1960 à 701 milliards de francs CFA en 1980 (MIPSP, 2008). L'un des objectifs visés de cette vision des gouvernants ivoiriens était de donner des emplois aux populations, de relever leur niveau de vie et d'envisager des socles édifiants pour le développement durable. En un mot, les structures agro-industrielles implantées dans les campagnes devraient y apporter le développement économique et social afin de contribuer au bien-être des populations riveraines.

Ce développement devrait se matérialiser à travers la réalisation des projets socio-économiques (écoles, centres de santé, routes, électricité, redevances annuelles, formation et encadrement des paysans etc.), pour parer à la paupérisation relative des communautés rurales. Malheureusement, plus d'un demi-siècle après l'indépendance du pays, le constat indique par endroit, une non-perceptibilité de ce développement dans les campagnes. Les zones d'exploitation semblent stagner dans leur dynamisme de développement et la dégradation des rapports sociaux entre industriels et autochtones semble s'aggraver (Babo, 2010). Tel fut le cas au Centre Ouest de la Côte d'Ivoire, au niveau du complexe sucrier de 
Zuénoula, où les populations Gouro des villages qui ont cédé des terres ont protesté pour promesses non tenues. Elles ont empêché la circulation des engins de la société vers l'usine de transformation de la canne à sucre (Baha-Bi, 1986). Un peu plus au Nord du pays, précisément dans les souspréfectures de Tafiré et de Badikaha, les chefs coutumiers et leurs populations ont organisé une manifestation de protestation contre les promoteurs de la société SUCAF-CI-2(AIP, 2013). L'explication de cette situation se trouve dans le fait que les clauses du contrat entre les populations ayant cédé 17.000 hectares de terres arables et la structure bénéficiaire n’ont pas été respectées par cette dernière.

Toupah, zone de la présente étude, compte les unités de production agricoles que sont la Société Africaine de Plantation d'Hévéa (SAPH) ; la Palme de Côte d'Ivoire (PALM-CI) et la PALMAFRIQUE. Après la signature des différents contrats de bail emphytéotique avec les autochtones Lodjoukrou dans les années 1960, ces entreprises ont réalisé des projets de développement socio-économique pour les populations ayant cédé leur terre. Mais depuis quelques années, les contrats sont remis en cause par les populations qui estiment insuffisants les réalisations en compensation des terres occupées par les structures. Quels sont les facteurs socioanthropologiques qui justifient le positionnement des acteurs dans le jeu du pouvoir ? Quelles sont leurs réelles motivations sociales, économiques et sociologiques, qui contrastent l'évolution du développement dans cette région rurale?

\section{Méthodologie}

Cette étude a eu lieu dans la sous-préfecture de Toupah dans le département de Dabou à environ 50km de la capitale économique Abidjan. Elle s'est déroulée sur une période de deux mois (02) allant de septembre à novembre 2015. La présente étude a mobilisé l'approche qualitative avec les entretiens libres et individuels dans l'optique de mieux cerné les questions en lien avec les structures agro-industrielles, les questions foncières et les dynamiques socio-économiques en contexte rural.

Au total une trentaine d'acteurs ont été interrogés dans les principes de la saturation des données et répartis comme suit : 10 jeunes, opposés au dispositif traditionnel en terme de l'héritage, 15 bénéficiaires dudit dispositif de succession et de l'acquisition de l'héritage et 05 personnes âgées, détentrices du savoir-faire, du savoir-être et gardiennes des valeurs culturelles ancestrales. Les données collectées auprès des différentes catégories susmentionnées ont fait l'objet d'analyse par le recours à la méthode dialectique et de l'analyse stratégique de Crozier. Ces méthodes combinées à la théorie socioconstructiviste telle qu'abordée par Baha-Bi (2001), Koné (2006) et Toh (2008) permettent de ressortir les enjeux de 
l'actualisation du dispositif traditionnel d'accès à la terre dans un contexte où les logiques traditionnelles et modernes semblent générer une superposition de droits à l'origine des rapports conflictuels.

\section{Résultats de l'étude}

L'enquête du terrain nous a permis d'aboutir à trois principaux résultats dans l'analyse de l'apport des structures agro-industrielles au développement de la commune rurale de Toupah. Ces trois points sont structurés autour du bilan de développement socioéconomique dans la sous-préfecture de Toupah, les antagonismes inhérents à la gestion des intérêts liés au foncier et les conflits subséquents.

\section{Implantation des structures agro-industrielles et actions de développement dans la région}

Implantées dans la sous-préfecture de Toupah en début 1960 avec l'intervention étatique, les structures agro-industrielles ${ }^{13}$ ont mené plusieurs actions d'appui au développement communautaire et de participation à la vie culturelle. Ces initiatives se résument à verser aux communautés villageoises des redevances annuelles en contrepartie des terres occupées, à faire des dons et des appuis en logistiques et en communication lors des cérémonies villageoises (dons les fêtes de génération, les décès, l'intronisation royale, etc.).

Les actions des structures ont permis aux populations de passer d'un habitat de type traditionnel à un habitat de type moderne. Les villages de Cosrou, Toupah, Kpanda et d'Irobo ont bénéficié d'électricité et d'eau courante. Au niveau du transport, l'entretien des routes et des pistes villageoises ont permis une fluidité routière, car des mini-bus desservent régulièrement Toupah et les villages environnants et les relient à Dabou chef-lieu de préfecture. Sur le plan éducatif et sanitaire, Toupah dispose d'un collège avec le premier cycle, de treize écoles primaires dont quatre écoles maternelles. Il ya aussi des centres de santé avec le personnel qualifié (médecin, infirmier, aides-soignants etc.).En dehors de ces actions, il faut aussi noter que les structures ont octroyé aux villageois, un jardin de bois de greffe (JBG) de trente (30) hectares. Cette initiative devrait permettre aux autochtones de se prendre en charge, et de faire face aux différents problèmes communautaires.

Enfin au niveau environnemental, les structures ont entrepris des actions de dépollution en créant des bacs lagunaires pour filtrer les eaux usées avant de les déverser dans la lagune. Les bordures des eaux sont

${ }^{13}$ Il s'agit de la Société Africaine de Plantations d’Hévéa (SAPH), de Palmier de Côte d'Ivoire (PALMCI) et de la société PALMAFRIQUE. On note également dans cette zone, la présence du Centre National de Recherche Agronomique (CNRA). 
pulvérisées avec des produits chimiques pour détruire les nids de prolifération des agents pathogènes, sources de maladies endémiques.

Une structure hôtelière a été créée en bordure de la lagune grâce aux structures. Et elle reçoit de nombreux citadins qui viennent pour la plupart passé le week-end en campagne, loin des pollutions de la ville. Au-delà, de toutes ces actions en faveur du développement dans la Sous-préfecture de Toupah, il faut noter que l'occupation des terres par les structures, reste une source de conflit entre les autochtones et les promoteurs en place. Mais comment ces conflits se manifestent ils empiriquement dans le champ social ?

\section{Conflits fonciers liés à la présence des structures}

Les structures implantées depuis 1960 dans la commune rurale de Toupah, ont signé des contrats de bail emphytéotique de quatre-vingt-dixneuf (99) ans avec les propriétaires terriens (Lodjoukrou). Et selon les données du Ministère de l'Agriculture (1997), ce sont au total plus de vingtcinq mille (25.000) hectares de terre arables qu'occupent ces structures dans cette région au sud de la Côte d'Ivoire. Ceci a entrainé selon le Ministère de l'Agriculture, la réduction de parcelles cultivables dans cette zone de 17\% en 1998 à 8\% en 2009. Ces données du Ministère indiquent une saturation foncière et un manque avancé de terres disponibles. L'enquête du terrain, nous a permis de constater une pression foncière, qui occasionne des conflits entre les membres d'une même famille (conflits intrafamiliaux), et des conflits entre responsables de structures et les villageois. Analysons le premier type de conflit, qui oppose les membres d'une même lignée familiale.

\section{Conflits intrafamiliaux}

Les parcelles sur lesquelles sont installées les structures agroindustrielles sont de la propriété des familles qui reçoivent en retour les redevances annuelles. Les redevances une fois perçues par les familles entrainent des conflits au sein de ces familles. Lorsque les gains financiers sont octroyés, ils sont automatiquement reversés aux neveux utérins, qui héritent du père défunt. L’application du matriarcat dans le partage des redevances est à l'origine de la fracture sociale de nombreuses familles à Toupah. Pour preuves, ce sont les enfants légitimes du défunt qui convoquent parfois les neveux au tribunal civil pour réclamer les biens laissés par leurs géniteurs. Mais contre toute attente, ils sont confrontés à la résistance des neveux qui refusent de revoir leur position.

Dans cette confrontation entre ayants droits coutumiers, et progénitures, les acteurs utilisent toutes sortes d'armes pour se nuire. Ce sont les menaces de morts, les agressions physiques, les envoutements et 
empoisonnements, enfin la pratique de la sorcellerie pour éliminer son adversaire.

Certaines plantations d'hévéa et de palmier à huile sont délaissées dans la broussaille, pour cause de conflit dans le lignage. A ce propos, un notable affirme que : "les conflits fonciers ont fait de nombreux morts dans nos villages à cause de cette pratique du matriarcat. Nous devons adapter nos coutumes aux réalités du moment, nos enfants souffrent beaucoup après notre mort ». Le constat empirique, dénote d'un rejet collectif du matriarcat au profit du patriarcat. Dans le discours des acteurs, l'on parle du respect des valeurs traditionnelles (maintien du matriarcat), mais dans les actes, de plus en plus de pères établissent des testaments en cachette pour garantir l'avenir de leurs enfants à leur mort. Ce qui engendre une opposition entre le droit moderne et le droit coutumier dans un espace social où la position des acteurs reste mitigée. Les conflits intrafamiliaux adossés aux intérêts fonciers, ont eu pour conséquences à Toupah l'apparition de l'individualisme. La fracture familiale a entrainé un nouveau mode de vie dans la société Lodjoukrou qui est axé sur le manque de solidarité parentale. Ce sera alors la montée de l'individualisme qui met en porte à faux les valeurs traditionnelles, qui prônent l'assistance mutuelle, la solidarité et le respect d'autrui. Selon le chef du village de Toupah : «Les familles ne sont plus soudées comme par le passé. Aujourd'hui chacun lutte pour son propre intérêt, et le bien être de chacun est une affaire individuelle ». L'enquête du terrain nous a aussi permis de constater l'effritement des valeurs de respect qui existaient entre cadets et aînés au sein des classes d’âge. Au fait, la classe d’âge des Mbedi (20-27 ans) refusent d'exécuter les décisions des anciens que sont les Nigbessi (60-67 ans). Ce manque de respect généralisé des cadets vis-à-vis des aînés au niveau des classes d’âge est symbolisé par les actes de grève et de revendication récurrente à l'encontre des entreprises implantées dans la région.

Ces cadets accusent leurs aînés d’avoir bradé leur patrimoine (terre) aux étrangers au point où le village se retrouve dans une situation de saturation foncière. "Nos parents sont la cause de notre souffrance dans le village. Ils ont loué toutes nos terres aux entreprises. Aujourd'hui, nous les jeunes sommes au chômage » déclarait un déscolarisé et sans emploi du village.

Dans la tradition Odjoukrou, les cadets sont dans l’obligation de demander la permission aux anciens avant tout acte collectif engageant le village. C’est après des cérémonies de libation pour implorer la bénédiction des ancêtres, que les Nigbessi (60-67 ans) donnent l'ordre aux jeunes de partir en guerre. Ce sont toutes ces rituels traditionnelles qui sont mises à mal par les cadets à Toupah et qui occasionnent des conflits 
intergénérationnels. Mais au fait, comment se passent les conflits qui opposent ces jeunes aux promoteurs?

\section{Conflits autochtones et promoteurs industriels}

Il existe plusieurs conflits entre autochtones et promoteurs industriels. Ces conflits sont souvent relatifs au non-respect des accords ou des principes du partenariat entre ces deux parties. Le plus souvent cela porte sur l'extension des activités de l'entreprise ou de la parcelle qu'elle occupe. Les responsables des structures dans leur volonté d'acquérir des nouveaux espaces pour l'extension de leurs exploitations sont souvent confrontés à une opposition de la part des autochtones. C'est donc ce qui a engendré le conflit de 2012 entre la palm ci et les autochtones des villages de Cosrou et d'Irobo. En effet selon T.K., président des jeunes de Cosrou : «... En 2012, la palm ci voulait occuper un espace sans l'accord des villageois. Selon les responsables de la structure, ils voulaient construire une usine pour la transformation de l'huile de palme. Lorsque les villageois l'ont appris, cela a provoqué leur mécontentement et ils ont barricadé les pistes menant à l'usine » le chef du village renchérit en disant: "Nous préférons donner ces parcelles à nos enfants déscolarisés qui ont décidé de revenir au village pour travailler la terre. Et cela pourra diminuer le nombre de chômeurs dans nos villages ».

En effet, plusieurs jeunes qui résidaient en milieu urbain sont revenus des villes pour s’installer dans les villages. Ces jeunes pour la plupart déscolarisés, ont besoin d'espace pour leurs exploitations. La présence de ceux-ci, ajoutée à celle des retraités revenus au village et des migrants a provoqué une saturation foncière. Du coup tous les espaces vaquant sont très convoités par l'ensemble des acteurs car chacun cherche un espace à exploiter. Les contrats signés entre autochtones anciens et promoteurs semblent déplaire considérablement aux autochtones cadets. Pour le signifier, ils les ont remis en cause.

\section{La remise en cause des contrats par les jeunes}

Les contrats ont été passés avec les ainés depuis les années 1960. Ces contrats sont aujourd'hui remis en cause par les jeunes générations qui les trouvent caduques. Ces contrats sont sources de conflits entres les jeunes générations et les structures agro industrielles. C'est donc ce que dit A.J.U., 32 ans, jeune déscolarisé, quand il affirme en ces mots : " ces structures ont passé des contrats avec nos parents, mais ces contrats datent de 1960. Les conditions dans lesquelles ces contrats ont été négociés ne sont plus d'actualité. Aujourd'hui les réalités de 1960 ne sont plus les mêmes.il convient donc de revoir ces contrats pour le bonheur de tous. Nous, ne pouvons pas accepter que des contrats passés depuis 1960, nous conduisent 
aujourd'hui, Nous ne pouvons pas nous conformer à cela. Quand on essaie de revendiquer nos droits, les structures ne veulent rien entendre. Nous aussi nous n'allons pas accepter ». Les conflits issus de ces revendications se résument en des griefs que les acteurs se reprochent mutuellement.

\section{Facteurs explicatifs des conflits selon les acteurs Griefs des villageois}

Les structures agro-industrielles présentent à Toupah, versent aux populations des compensations financières qui s'élèvent à 44 millions de francs CFA. Ces redevances villageoises depuis 1960 jusqu'à 2001 n’ont pas connu d'augmentation. A partir de 2001ces redevances connaitront une hausse en passant de 44 millions à 60 millions de francs CFA (DCS-2015). Malgré la hausse qu'a connue la redevance villageoise, les populations estiment qu'elle n'est pas suffisante. En effet selon elles, la population de Toupah sur le plan démographique, a connu une importante augmentation de 1960 jusqu'à ce jour. Cette population est passée de 8000 habitants en 1998 (RGPH-1998) à 30000 habitants en 2014 (RGPH-2014). Les autochtones estiment qu'au vue de cette augmentation des populations, les compagnies Agro-industrielles devraient revoir les redevances à la hausse car selon elles, l'augmentation des populations entraine l'apparition de nouveaux besoins et par conséquent les redevances devraient également subir une hausse.

Selon A.T.K, un cadre du village : "Voyez-vous ces structures sont sur nos terres depuis 1960. Elles occupent ces terres et en tirent beaucoup de bénéfices. Mais en retour la redevance qu'elles doivent verser à la population ne change pas. Depuis 1960, c'est en 2001 qu'ils ont augmenté un peu et depuis lors plus rien. Or la population qui était là en 1960, n'est plus la même qu'aujourd'hui. La population a augmenté. Les structures là devraient tenir compte de tout ça. Mais ce n'est pas leur problème».

Au-delà de la redevance villageoise les populations accusent les structures d'abandonner les infrastructures routières et de service, et de ne pas donner de l'emploi à leurs enfants. C'est ce que dit L.L.J., membre du conseil villageois : "Tu vois ces structures sont avec nous ici et nous n'avons pas de routes. Toutes nos routes sont dégradées, dans le village les poteaux électriques ne fonctionnent plus et le village est plongé dans le noir. Ces structures sont présentes dans le village et les jeunes du village n'ont pas d'emploi. La majorité des jeunes est au chômage, tout ce qu'elles leur offrent ce sont des contrats temporaires. Que peut faire un salaire contractuel. Ils ne sont jamais embauchés pour espérer quitter la précarité, vous voyez tout ça. Or, nous avons signé un contrat gagnant-gagnant avec toutes ces structures mais on constate que c'est elles seules qui profitent et 
pas nous». Toute cette situation concourt à altérer les rapports entre les populations et les structures présentes sur leur sol.

Au-delà du manque d'opportunité d'emploi, les villageois reprochent aux structures la précarité de l'emploi qu'elles offrent aux minorités autochtones embauchés. Cette minorité, selon eux est employée au titre de contractuels saisonniers. Et pour eux, cela ne garantit aucune protection sociale satisfaisante. En dehors de la qualité de l'emploi, ils évoquent le faible revenu de ces embauchés autochtones, qui touchent des salaires qui ne correspondent pas aux difficultés des tâches. Ils dénoncent la passivité des promoteurs face à la restauration des infrastructures sociales (routes, écoles, dispensaires etc.), qui tombent en ruine par l'usure du temps.

Selon ce cadre et fils de Toupah : «Aujourd'hui, il est quasiment difficile de circuler dans la région pour cause de pistes et routes dégradées. Certains villages de la Sous-préfecture Toupah ne voient les mini-bus qu'une fois par semaine. A cause de l'état des pistes les transporteurs refusent de s'y rendre ".

Ce manque de suivi au niveau des infrastructures routières semble révolter les villageois, qui disent que dans les clauses du contrat, les structures s’étaient engagées à faire de la maintenance pour faciliter la fluidité routière, et l'accessibilité des zones les plus reculées de la commune.

Pour ce jeune natif de d'Irobo : «Au début du contrat, les structures donnaient l'eau courante et l'électricité gratuitement aux villageois. Mais de nos jours seule l'eau est gratuite, nous payons nos factures d'électricité à la CIE de Dabou, comme tout le monde. Ce n'est pas normal ».

Le non-respect des clauses des contrats semble pousser les villageois à les remettre en cause, pour que le partenariat gagnant-gagnant puisse avoir tout son sens.

Enfin, ils déplorent les violences et agressions qui leur sont infligés par les forces de l'ordre à chaque fois qu'ils manifestent pour réclamer aux structures la rétrocession de leurs terres. Et les promoteurs, quels sont leurs griefs ?

\section{Griefs des responsables de structures}

Quant aux responsables des structures Agro-industrielles, ils reconnaissent être dans un partenariat gagnant-gagnant avec les villageois, mais déplorent certains de leurs actes, qui selon eux ralenti leur production au plan national et international. Pour ces promoteurs, les autochtones sont eux-mêmes à la base de leurs problèmes car l'organisation mise en place par ceux-ci privilégient la recherche d'intérêts individualistes plutôt que communautaires. 
En effet, pour la gestion de la redevance villageoise, les populations ont installé un comité de gestion de cette redevance. Dans la pratique de ce comité, certaines anomalies ont été observées dans la gestion des fonds collectifs. Et cette situation a engendré une crise de confiance au sein des villageois selon les promoteurs. D'un commun accord, les villageois ont décidé de dissoudre ce comité de gestion et confié la gestion des redevances au Sous-préfet. Les promoteurs disent ne pas comprendre pourquoi, les villageois les accusent de remettre leurs fonds à ce comité jadis homologué à les recevoir.

Pour le Directeur du centre de la SAPH Toupah: «Nous payons chaque année la redevance villageoise aux populations. Les comités de gestion privilégient les intérêts individuels au détriment des intérêts collectifs. Aucun compte ne leur est demandé et c'est à nous que les populations s'en prennent ».

Les responsables de structures déplorent ce comportement qui selon eux ne peut dynamiser le développement de la région. Ils disent donner ces redevances aux villageois pour qu'ils puissent se prendre en charge, et entreprendre des actions de développement pour leur région. Ils affirment donc ne pas comprendre en quoi ils peuvent être responsables de cette mauvaise gestion.

En dehors de cela, les promoteurs dénoncent l'attitude de défiance des jeunes autochtones lors de grèves et des protestations. Les actes de sabotage et d'intimidation quotidiens des jeunes sont liés selon eux à leurs comportements indélicats qui se résument à une grande estime de soi, à un sentiment prononcé de l'autochtonie etc. La nature des rapports sociaux semble déterminante dans la problématique du développement de Toupah commune, dans la mesure où elle traduit le degré de cohésion sociale. Cette cohésion sociale semble être un socle édifiant pour que le développement puisse être total à Toupah.

\section{Discussion des résultats}

Kanga (2002) dans son rapport sur les innovations en milieu rural, évoque que traditionnellement en Afrique, la terre était un bien collectif de tous les habitants. Chaque individu selon cet auteur pouvait recevoir en jouissance une parcelle du patrimoine communautaire. Les soumissions aux règles communautaires ont longtemps maintenu une relative cohésion sociale et préservé la crise foncière. L'auteur soutient que l'adoption et la diffusion des innovations par le biais des entreprises agro-industrielles en milieu rural, confère à ce lieu un regain de développement social et économique. Il fait une analyse sous l'angle évolutionniste pour dire que le développement rural permet de faire passer les populations rurales de leur mode de vie traditionnel au mode de vie moderne et de consommation à l'image des 
sociétés dites développées. Quant à Durand (2008), il soutient que les défis auxquels sont confrontées toutes les politiques de développement sont liés pour la plupart aux mauvaises pratiques des instances de contrôle de l'accès aux ressources. Il contredit les outils de régulation à partir de leurs failles et de leurs lacunes, eu égard aux nombreux conflits qui secouent les milieux ruraux, après implantation des structures agro-industrielles. Pour Marc (2002), les causes des conflits fonciers dans les milieux ruraux, après adoption des sociétés agro-industrielles, relèvent de la difficulté de cohabitation entre le droit coutumier et le droit moderne.

Aucun de ces auteurs n'a relevé les bouleversements des mécanismes et des fondamentaux de la société traditionnelle après implantation des structures agro-industrielles. Ce qui fait la particularité de l'enlisement des valeurs coutumières chez les Lodjoukrou, c'est que ce sont les acteurs euxmêmes, qui sont originaires du village qui détruisent lentement ces deux piliers de leur tradition (matriarcat et classes d'âge) pour des enjeux économiques liés au foncier. Mais il faut dire que ce phénomène est apparu dans le village, après l'adoption des structures agro-industrielles dans la région.

En effet, la société Odjoukrou est caractérisée par son lien fort à la filiation matrilinéaire et aux classes d'âge. A travers le matriarcat, ce peuple attribue l'héritage au neveu utérin, qui se doit d'assister la veuve et les enfants de l'oncle défunt. Mais pour des questions d'intérêts individuels, nombreux sont les neveux qui n'accomplissent plus ce devoir d'assistance aux enfants de l'oncle défunt. Et les fréquents conflits enregistrés dans la région, amènent lentement les sujets à se munir de testaments en vue de préserver la survie de leurs progénitures, après leur mort. Cet état de fait, tend à faire disparaitre petit à petit le matriarcat, qui ne doit son salut qu'à un nombre réduits de conservateurs dans le village. Donc depuis que les structures agro-industrielles se sont installées les enjeux socio-économiques sur le foncier ont contribué à diviser considérablement les membres d'une même famille, notamment les neveux et les enfants déshérités. Ce conflit intrafamilial a dénaturé le matriarcat, qui au lieu de souder les fibres de fraternité entre segments familiaux, est considéré de nos jours par la majorité des villageois, comme un phénomène comportant des germes conflictuels. La confection en secret de nombreux testaments par les villageois tue lentement le matriarcat, qui devient insignifiant petit à petit aux yeux des acteurs. "Nous devons préserver la survie de nos enfants après notre mort. Le matriarcat est révolu » déclarait E.P. un cadre originaire de Toupah.

Les cadets du village négligent les lois qui caractérisent les classes d'âge. Leurs comportements indélicats vis-à-vis des aînés trouvent leur justification dans les enjeux socio-économiques sur le foncier à Toupah. Les classes d'âge chez les Odjoukrou sont fondées sur des valeurs comme la 
solidarité, l'entraide et l'assistance mutuelle en cas de danger. Aujourd'hui, toutes ces lois et valeurs sont presqu'inexistantes dans la coutume, car le conflit intergénérationnel perdure et les classes d’âge sont en train de subir de profonds changements. L'effondrement de ces deux institutions traditionnelles trouve sa justification dans les conflits d'intérêts qui opposent les acteurs sur le foncier. Comment comprendre que les socles de la tradition se meurent au profit de la course aux intérêts individuels?

Les responsables industriels de Toupah utilisent la stratégie de l'approche individuelle pour affaiblir les crises sociales dans le village, en vue de préserver leurs acquis dans le jeu du pouvoir. Certains acteurs clés dans les instances de décision sont détournés discrètement pour jouer le jeu des entreprises. On leur fait des propositions alléchantes et ils sont des espions à la solde des promoteurs. Choisis parmi les adultes et parfois parmi les jeunes, ces individus à double visage informent les promoteurs à chaque fois qu'une révolte se prépare. Le reste de la communauté ignore leur existence, mais ils sont connus par l'autorité Sous-préfectorale. Selon le Sous-préfet: "C'est un bois sacré constitué d'individus originaires de Toupah, que les structures emploient en quelque sorte dans la discrétion pour pouvoir travailler sereinement. Ce groupe est un vrai palliatif de crise pour la région ».

On cherche à expliquer la construction des règles (le construit social) à partir du jeu des acteurs empiriques, calculateurs et intéressés. Ces acteurs sont dotés de rationalité, même si elle est limitée ; ils sont autonomes et rentrent en interaction dans un système qui contribue à structurer leurs jeux. Les acteurs interviennent dans un système, l'organisation, qui doit et peut s'ajuster à des contingences et des changements de natures diverses.

Ce «jeu d'alliances discret», comme le dit Crozier, se présente comme l'élément catalyseur de l'embrasement de l'organisation. L'analyse stratégique confronte alors les acteurs et leurs objectifs. Ni les uns, ni les autres n’ont de rôle figé, d’où l’idée de système basé sur les interrelations. Les acteurs sont des individus ou des groupes. Les objectifs sont explicites ou non et sont multiples. Pour résoudre les problèmes concrets, il faut raisonner au cas par cas.

Cette stratégie vise à briser l'élan des grèves et des revendications de droits de propriété foncière organisées par les jeunes qui sont au premier rang des conflits. Le groupe des contestataires est scindé en deux, et la divergence des idées fini par mettre fin à la crise. Le développement socioéconomique des milieux ruraux constitue un axe prioritaire sur lequel l'Etat doit attacher une attention particulière afin de prévenir les conflits qui sont une entrave à la cohésion sociale. 


\section{Conclusion}

Au terme du présent travail, convient-il de dire que les structures agro-industrielles implantées dans les localités rurales donnent aux populations un espoir de développement. Cet espoir pose la question fondamentale de l'impact de ces structures de développement dans l'amélioration des conditions de vie des populations rurales. Ces populations souvent spoliées de leurs biens fonciers, sont confrontées à des problèmes structurels de bien-être et de reproduction sociale. Le cas de la commune rurale de Toupah, nous laisse voir des contradictions liées à la présence des structures agro-industrielles dans les zones rurales. Ces structures semblent épouser les théories économistes et matérialistes de développement pour satisfaire les populations paysannes, qui bien qu'acceptent ces visions sont dans une logique sociale fondée sur la perceptive immatérielle du développement.

De fait, ces structures agro-industrielles orientent leurs actions dans la réalisation d'infrastructures socio-économiques, mais elles semblent occulter du coup l'aspect humain fondé sur la qualité des rapports sociaux, la responsabilité des populations, et la prise en compte des référents culturels et des aspirations essentielles des populations. Ces visions contradictoires de développement sont souvent à l'origine des conflits sociaux observés entre populations elles-mêmes et entre villageois et promoteurs. Ces différents conflits mettent en mal le bien-être des villageois et la performance de l'industrie dans notre pays. C'est pourquoi, la prise en compte de toutes ces visions dans une articulation constructive pourrait favoriser le développement des zones où sont présentes ces structures. Dans l'optique d'une intégration harmonieuse des structures agro-industrielles dans les campagnes en Côte d'Ivoire.

\section{References:}

1. Agence Ivoirienne de Presse (2013), Rapport sur les litiges fonciers au nord de la Côte d'Ivoire. Novembre.

2. Babo, A. (2010), Conflits fonciers, ethnicité politique et guerre en Côte d'Ivoire, Paris : Alternative sud, Vol. 17, pp. 95-118.

3. Baha Bi, Y. (1986), L'impact socio-économique de la SODESUCRE sur le département de Zuénoula au centre de la Côte d'Ivoire. Thèse de doctorat $3^{\text {ème }}$ Cycle, Abidjan: Université d'Abidjan, Institut d'Ethno-Sociologie.

4. Colin, J.P. (1990), La mutation d'une économie de plantation en basse Côte d'Ivoire. Paris : Edition ORSTOM.

5. Chauveau, J.P. et Koffi, K.J. (2005), Question foncière et construction nationale en Côte d'Ivoire: enjeux silencieux d'un 
coup d'Etat. Montpellier: Institut de Recherche pour le Développement.

6. Crozier, M. et Friedberg, E. (1977), L'acteur et le système. Les contraintes de l'action collective. Paris : Edition du Seuil, collection Point Essais.

7. Direction régionale de l'éducation nationale (2015), Taux de fréquentation des écoles primaires à Toupah commune. Rapport.

8. Direction régionale de la santé publique et de la lutte contre le sida (2015). Taux de fréquentation des centres de santé dans la souspréfecture de Toupah. Dabou : Région des Grands Ponts.

9. Durand, L. (2008), Gérer le foncier rural en Afrique de l'Ouest: dynamiques foncières et interventions publiques. Paris: Edition Karthala, URED.

10. Direction régionale de l'éducation nationale (2015), Effectif des élèves de la Sous-préfecture de Toupah. Rapport. Avril.

11. Institut National de la Statistique (2015). Recensement Général de la Population et de l'Habitat - 2014. Côte d'Ivoire.

12. Koffi, K.J. (2006), Analyse économique de l'aménagement forestier dans une perspective de développement socialement durable en Côte d'Ivoire. Thèse de doctorat en sciences économiques. Montpellier : Université de Montpellier I.

13. Koné, L. (2014), Problématique de la participation des allogènes au développement socio-économique de la sous-préfecture de Toupah. Abidjan : Institut d’Ethno-Sociologie, Université Félix HouphouëtBoigny.

14. Koné, M. (2006) : Foncier rural, citoyenneté et cohésion sociale en Côte d'Ivoire : la pratique du tutorat dans la sous-préfecture de Gboguhé, in Actes du colloque international «Les frontières de la question foncière ».

15. Marc, B. (2002), Conflits fonciers en Afrique central. Paris : Edition clair, VFR.

16. Ministère de l’Agriculture et du développement rural (2013), Etat du foncier en Côte d'Ivoire. Rapport. Abidjan.

17. Ministère de l’Agriculture et du développement rural (2014), Etat des baux emphytéotiques ruraux. Abidjan.

18. Ministère de l'Industrie et de la Promotion du Secteur Privé (2008), Rapport sur l'industrie ivoirienne. Abidjan.

19. Ogni, K.B. (2002), Rapport sur l'innovation et le transfert de technologie en milieu rural ivoirien. Abidjan: Institut d'EthnoSociologie, Université d'Abidjan.

20. SAPH (2015), Rapport de la réunion avec les villageois. DCAI, Toupah. Juin. 
21. SAPH (2015), Gestion des redevances annuelles. DCAI, Toupah. Rapport. Mai.

22. Toh, A. (2008), Conflits fonciers, gouvernance locale et dynamismes sociaux de régulation dans le sud-est ivoirien : étude de cas de la sous-préfecture de Bonoua. Thèse de doctorat unique, Abidjan : Institut d'Ethno-Sociologie, Université d'Abidjan.

23. Toh, A. (2011), Evolution des dispositifs d'accès à la terre et crise du lien social en milieu rural ivoirien. Revue ivoirienne d'anthropologie et de sociologie. KASA BYA KASA, $\mathrm{n}^{\circ}$ 20, pp. 50-90.

24. Zongo, M. (2009), Terre d'Etat, loi des ancêtres ? Les conflits fonciers et leurs procédures de règlement dans l'Ouest du BurkinaFaso. Cahier du CELESH, Tome XXIV, N³3.

25. Sawadogo, A. (1977), L'agriculture en Côte d'Ivoire. Paris : Presses universitaires de France. 